\title{
Optimization of bidirectional nozzle of crude oil storage tanks based on CFD approaches
}

\author{
JiaJun Ye ${ }^{1, a}$, GenMin Zhu ${ }^{* 1,2,4}$, Jun Lyu³ , XiaoHui Zhang1, MingQiang Zhang1, \\ JianKang Shen ${ }^{1}$
}

${ }^{1}$ School of Petrochemical and Energetic Engineering,Zhejiang Ocean University, Zhoushan 316022, China

\begin{abstract}
${ }^{2}$ United National-Local Engineering Laboratory of Harbor Oil\&Gas Storage and Transportation Technology, Zhoushan 316022, China
\end{abstract}

${ }^{3}$ School of Naval Architecture and Mechanical-electrical Engineering, Zhejiang Ocean University, Zhoushan 316022,China

ahuobiterr@163.com

Key words: crude oil storage tanks, jet flow, bidirectional nozzle, CFD

Abstract. In order to study the influence of the nozzle parameters of the rotating jet agitator on the mixing effects, the CFD software is used to simulate the flow field of the bidirectional nozzle of a rotating jet agitator in large crude oil tanks. The mass flow, turbulent kinetic energy and flow velocity are analyzed and compared when nozzles with different diameters and lengths. Finally, the nozzle with the best jet performance is optimized. The results show that the acceleration of the long nozzle is better than the short nozzle, but the long nozzle will cause the loss of the turbulent kinetic energy, which needs to be considered in the actual equipment selection process. Although the large diameter nozzle has high mass flow, the uneven distribution of velocity in the tube will result skewed jet flow.

\section{Introduction}

In the petrochemical industry, there are many devices running after a long period of operation will produce scale and solid deposition ${ }^{[1]}$. Therefore, the cleaning operation has gradually become an indispensable industry in petrochemical field, and the cleaning effects directly affects the operation and working efficiency of the equipment. At present, commonly used cleaning methods mainly include chemical cleaning and physical cleaning. Chemical cleaning is more complex, with large investment and great harm to the environment ${ }^{[2-4]}$. However, high pressure water jet cleaning of physical cleaning method is gradually favored by people with its unique advantages. The high pressure water jet cleaning technology uses high pressure generator to produce high pressure water, and then use the nozzle to change the pressure into a highly aggregated water jet flow, and impact the surface of the cleaning material, so that the adhesion layer is separated from the surface of the matrix to achieve the cleaning process ${ }^{[5-7]}$. The nozzle is a very important part of the high pressure water jet cleaning system, which affects the other parts of the cleaning system ${ }^{[8]}$. The study adopts the method of CFD numerical simulation to establish the flow field of bidirectional nozzle, and a series of modified nozzles are established by changing parameters of the nozzle. The influence of the nozzles' diameters and lengths on the flow field are compared and analyzed, and the best jet nozzle is selected. 


\section{Computational methods}

Governing Equations. The governing equations for turbulent flow in the present study are the continuity equations for mass conservation and unsteady Reynolds-averaged Navier-Stokes equations for momentum transport, as follows:

$$
\begin{aligned}
& \frac{\partial \mathrm{u}_{i}}{\partial x_{i}}=0, i=1,2,3 \\
& \rho \frac{\partial u_{i}}{\partial t}+\rho u_{j} \frac{\partial u_{i}}{\partial x_{i}}=\rho F_{i}-\frac{\partial p}{\partial x_{i}}+\frac{\partial}{\partial x_{j}}\left(\mu \frac{\partial u_{i}}{\partial x_{j}}-\rho \overline{u_{i}^{\prime} u_{j}^{\prime}}\right), i, j=1,2,3
\end{aligned}
$$

Where $\mathrm{i}=1,2,3$ represents $\mathrm{X}, \mathrm{Y}, \mathrm{Z}$.direction respectively. $-\overline{\rho u_{i} u_{j}}$ is the Reynolds stress tensor, $u$ is the averaged velocity, $\rho$ is the density,in the present study.

There are several turbulence models employed to satisfy the governing equations in this similar study.The standard $k-\varepsilon$ model are chosen for use in this study. Transport equations of $k$ and $\varepsilon$ in standard $k-\varepsilon$ model as:

$$
\begin{aligned}
& \frac{\partial(\rho k)}{\partial t}+\frac{\partial\left(\rho k u_{i}\right)}{\partial x}=\frac{\partial}{\partial x_{i}}\left[\left(\mu+\frac{\mu_{t}}{\sigma_{k}}\right) \frac{\partial k}{\partial x_{j}}\right]+G_{k}+G_{b}-\rho \varepsilon-Y_{m} \\
& \frac{\partial(\rho k)}{\partial t}+\frac{\partial\left(\rho \varepsilon u_{i}\right)}{\partial x}=\frac{\partial}{\partial x_{j}}\left[\left(\mu+\frac{\mu_{t}}{\sigma_{\varepsilon}}\right) \frac{\partial \varepsilon}{\partial x_{j}}\right]+C_{1 \varepsilon} \frac{\varepsilon}{k}\left(G_{K}+C_{3 \varepsilon} G_{b}\right)-C_{2 \varepsilon} \rho \frac{\varepsilon^{2}}{k}+S_{\varepsilon} \\
& G_{k}=\mu_{t}\left(\frac{\partial u_{i}}{\partial x_{j}}+\frac{\partial u_{j}}{\partial x_{i}}\right) \frac{\partial u_{i}}{\partial x_{j}}
\end{aligned}
$$

Gk is the turbulent kinetic energy production caused by an average velocity gradient, Gb is the turbulent kinetic energy production caused by buoyancy, YM represents the effects of fluctuating expansion of turbulent on dilatation dissipation in compressible turbulent, $C_{1 \varepsilon}, C_{2 \varepsilon}$ and $C_{3 \varepsilon}$ are constant, $\sigma_{k}$ and $\sigma_{\varepsilon}$ are turbulent Prandtl number of turbulent kinetic energy $\mathrm{k}$ and turbulent dissipation rate $\varepsilon, S_{k}$ and $S_{\varepsilon}$ are the user-specified source term.

The free surface is modeled by using Volume-of-Fluid (VOF) approach and a high-resolution interfacecapturing scheme, as (6).

$$
\frac{\partial \alpha}{\partial t}+v \cdot \nabla \alpha=0
$$

Physical Models and Computational Domain. In the present study, the basic dimensions of the nozzle model refer to the common tank cleaning bidirectional jet nozzle parameters, and its shape and structure are shown in the Fig.1. And the inner region of the tube and the efflux generating area of the outlet are selected as the computational domain. The tube wall was set as the wall surface, and the inlet is set as pressure boundary with pressure is $1 \mathrm{Mpa}$.The pressure boundary is also set at 
outside the jet area.In terms of physical conditions, light crude petroleum with a density of $875 \mathrm{~kg} / \mathrm{m} 3$ was selected as the liquid phase, and incompressible air was selected as the gas phase.the computational domain and the boundary conditions is shown as Fig.2.

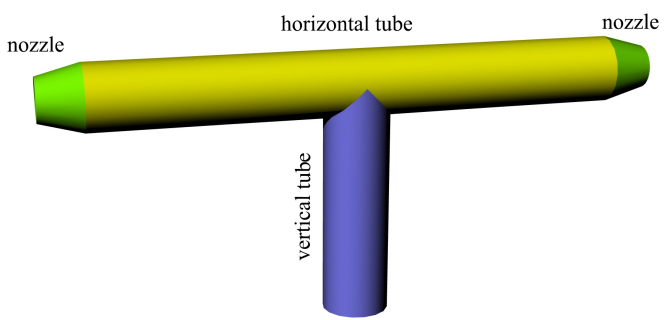

Figure 1. Bidirectional nozzle structure diagram

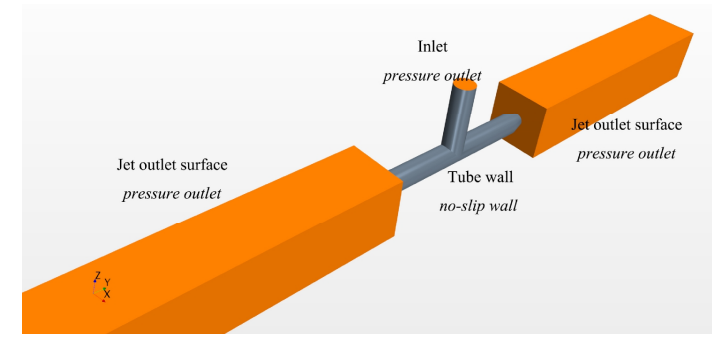

Figure 2. Calculate domain and boundary conditions

Study On Grid Convergence. The study choose polyhedron meshing calculation domain, near the tube wall uses the prism layer encryption. In this study, 5 grids are divided, with the number of grids ranging from 0.2 million to 0.85 million. The average outlet velocity iswas taken as the reference standard for grid dependency analysis.Its calculation results as shown in Fig.3, the results difference is small but gradually increased along with the increase in grid average outlet velocity and stableilized after the 0.41 million grid, so choose 0.41 million grid for subsequent calculations.The 0.41 million grid is shown in the Fig.4.

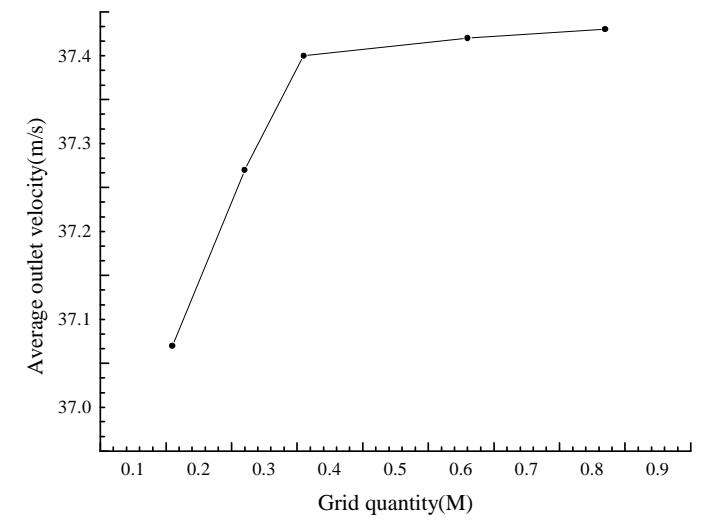

Figure 3. Grid dependency calculation results

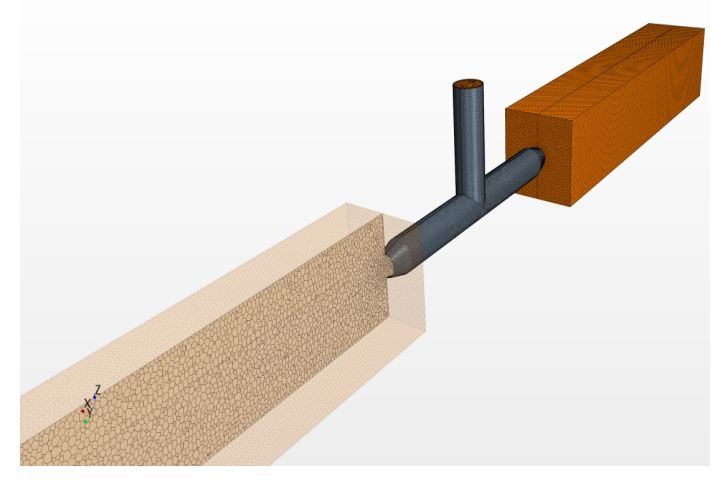

Figure 4. 0.41 million grid

\section{Numerical results and discussion}

In this paper, different diameters and lengths of nozzles are selected to improve the prototype nozzle. The eight modification parameters are shown in Table 1. Through numerical simulation, eight modifications are calculated, and the results are analyzed and optimized.

Table 1 Modified nozzle parameters

\begin{tabular}{cccc}
\hline Type & Diameter $(\mathrm{cm})$ & $\begin{array}{c}\text { Caliber } \\
\text { (Length/Diameter) }\end{array}$ & Length(cm) \\
\hline 1 & 10.5 & 0.5 & 5.25 \\
2 & 10.5 & 1.0 & 10.50 \\
3 & 10.5 & 1.5 & 15.75 \\
\hline
\end{tabular}




\begin{tabular}{cccc}
\hline 4 & 11.0 & 0.5 & 5.50 \\
5 & 11.0 & 1.0 & 11.00 \\
6 & 11.0 & 1.5 & 16.50 \\
7 & 11.4 & 0.5 & 5.70 \\
Prototype & 11.4 & 1.0 & 11.40 \\
8 & 11.4 & 1.5 & 17.10
\end{tabular}

The Calculation Results of Eight Different Modified Nozzles' Mass Flow. The calculation results of eight different modified nozzles' mass flow are shown in Fig.5. From Fig.5, it can be seen that with the increase of the nozzle length, the outlet flux increases obviously. The mass flow of the 1.5 times of the diameter is the greatest, the nozzle length has influence on the outlet flux, and the long nozzle jet performance is better than the short one.

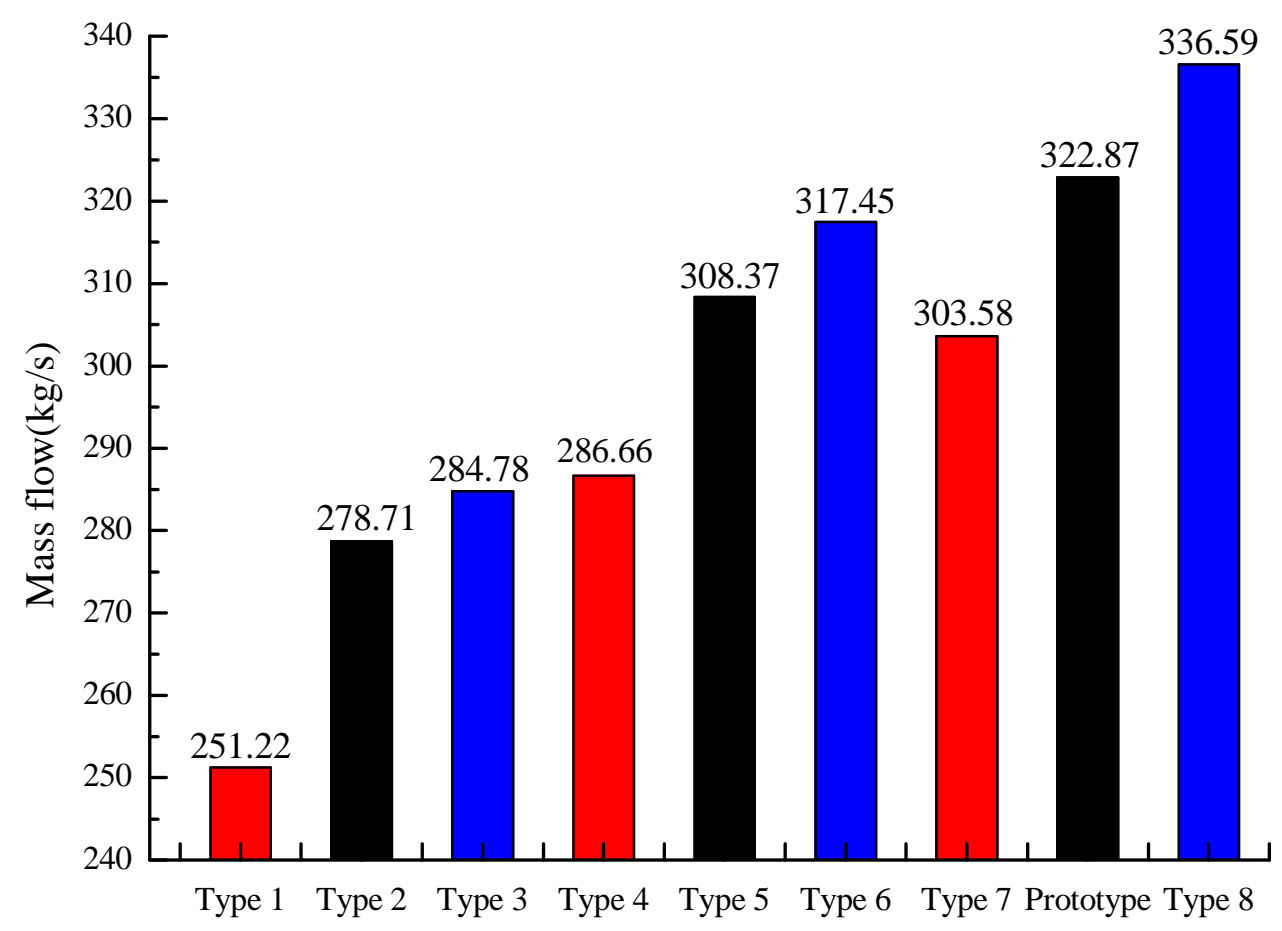

Figure 5. Calculation results of eight different modified nozzles' mass flow

The Calculation Results of Eight Different Modified Nozzles' Average Turbulent Kinetic Energy. The calculation results of eight different modified nozzles' average turbulent kinetic energy are shown in Fig.6. It can be seen from Fig.6 that the turbulent kinetic energy of the short nozzle is greater with the same diameter, while the turbulent kinetic energy of the large diameter nozzle with the same caliber is greater than that of the small diameter nozzle. This is due to the change of the wall surface caused by the change of the length and circumference of the nozzle. It can be seen that 1.5 times caliber nozzle's turbulence kinetic energy loss is greater than 1.0 times caliber nozzle, while the turbulent kinetic energy effects of the nozzles with 0.5 to 1 times caliber is similar. From the perspective of turbulent kinetic energy, long nozzle is not a good choice. 


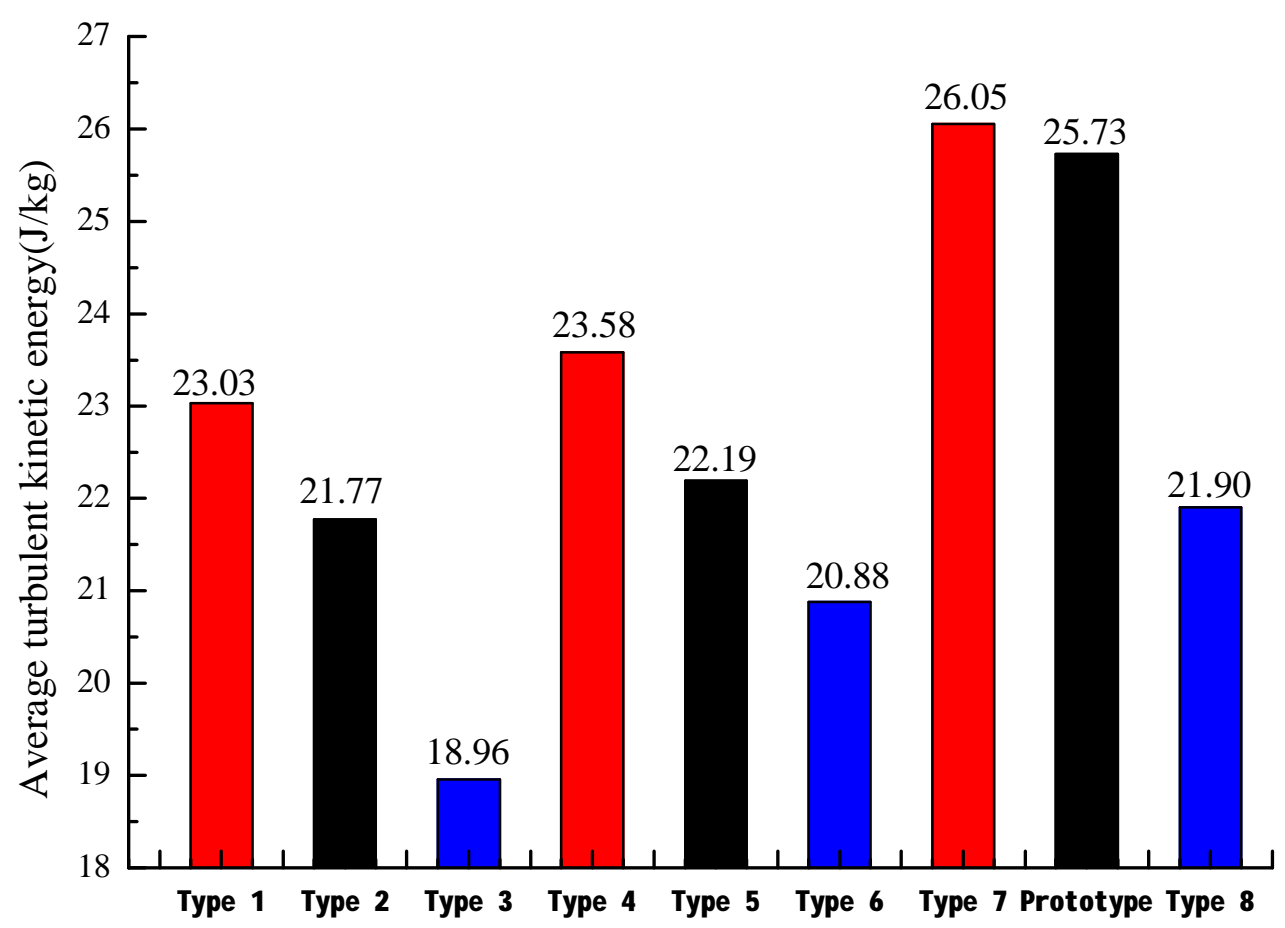

Figure 6. Calculation results of eight different modified nozzles' average turbulent kinetic energy

The Calculation Results of Eight Different Modified Nozzles' Average Velocity. The calculation results of eight different modified nozzles' average velocity are shown in Fig.7. It can be seen from Fig.7 that the long nozzle has a great advantage in the flow velocity. The longer contraction section accelerates the convergence of the flow field. This can from one side to explain why the mass flow of the long nozzle is higher than the short nozzle. It can be seen that the small diameter nozzle's flow velocity is faster than the large diameter nozzle under the same caliber, so that the small diameter nozzle has great effects on converging flow field.

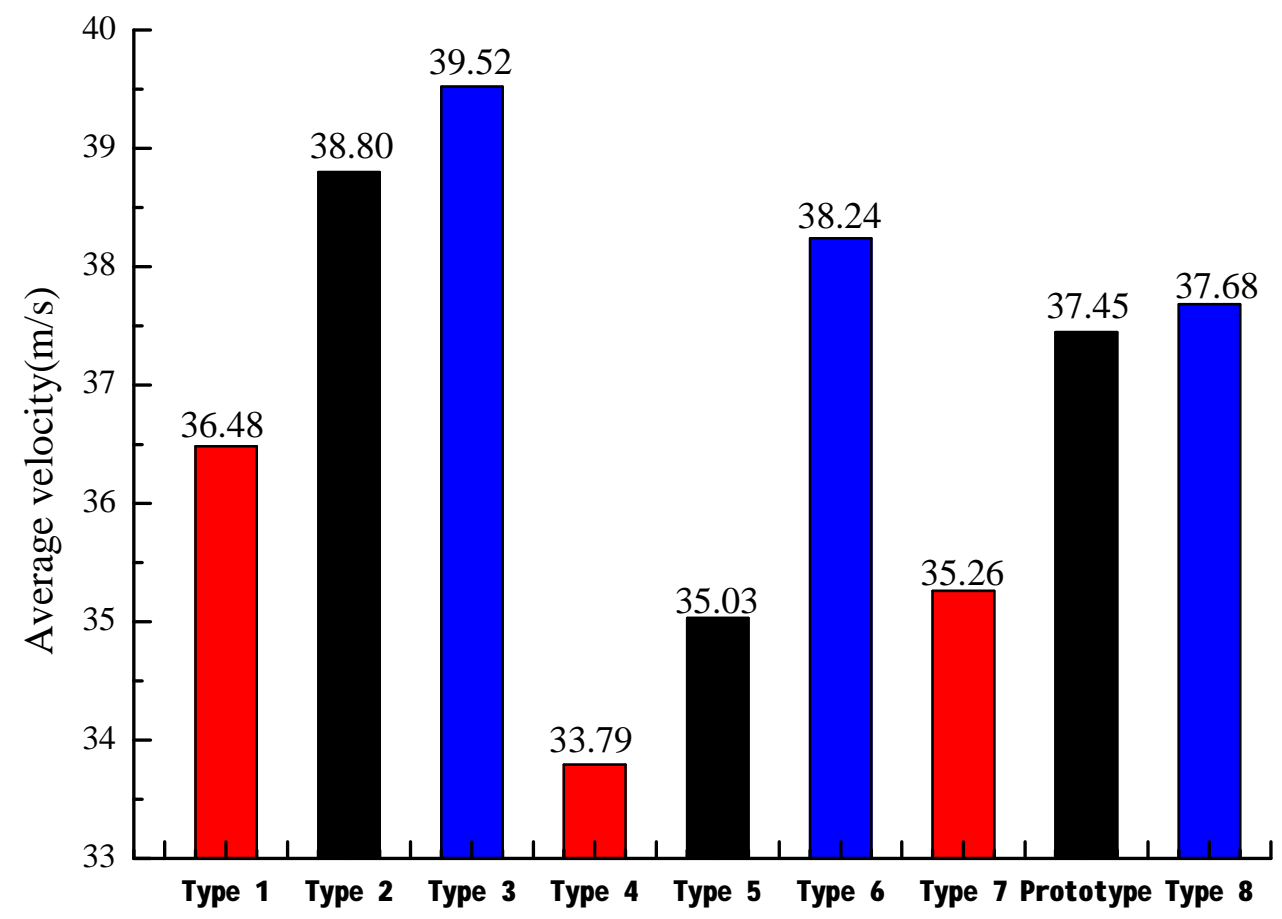

Figure 7. Calculation results of eight different modified nozzles' average velocity

The Velocity Distribution of Eight Different Modified Nozzles. The velocity distribution of eight different modified nozzles are shown in Fig.8. From Fig.8, it can be seen that the type 3 
nozzle has an obvious jet bifurcation, showing that the nozzle's flow velocity is uneven. In the actual process, the flow diffusion will cause the loss of energy, that is, partial fluid may not be washed to the surface of the cavern and be wasted. In the rest of the nozzles' jet velocity in the figure have large area, including type 1 and type 2 have large high velocity area, and the maxmum velocity is even more than $50 \mathrm{~m} / \mathrm{s}$. The fast velocity is very important for flushing oil sludge. So, the most important reference coefficient of the nozzle is flow velocity.

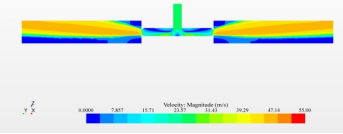

(a)Type 1

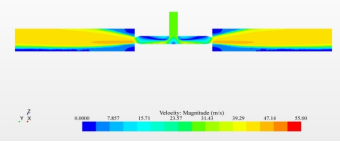

(e)Type 5

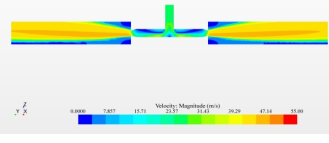

(b)Type 2

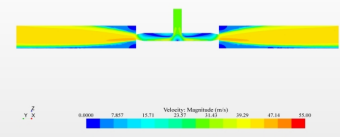

(f)Type 6

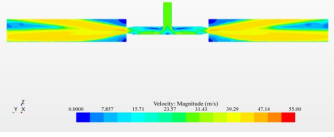

(c)Type 3

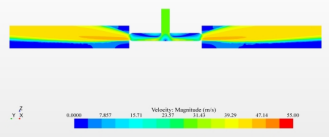

(g)Type 7

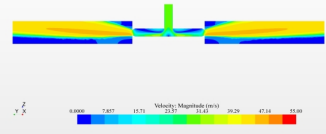

(d)Type 4

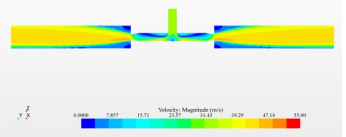

(h)Type 8

Figure 8. The velocity distribution of eight different modified nozzles

The liquid volume fraction distribution of eight different modified nozzles. The liquid volume fraction distribution of eight different modified nozzles are shown in Fig.9. It can be seen that the short nozzle's jet angle is not parallel to the Y axis, the liquid fills all the space in the tube, indicating that the direction of fluid flow velocity is still not parallel to the $\mathrm{Y}$ axis, which has a great influence on the jet range and the impact velocity.

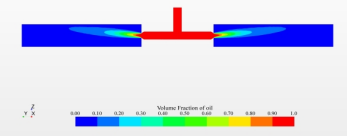

(a)Type 1

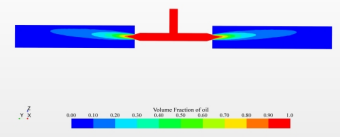

(e)Type 5

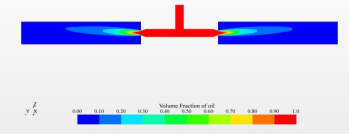

(b)Type 2

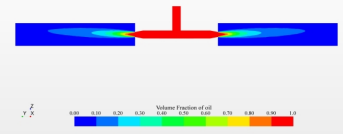

(f)Type 6

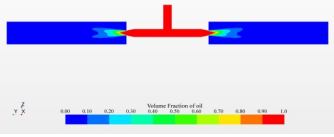

(c)Type 3

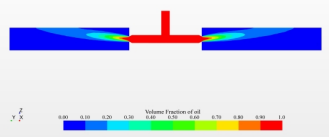

(g)Type 7

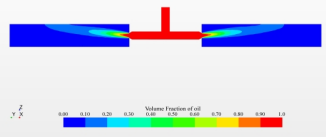

(d)Type 4

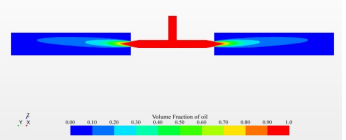

(h)Type 8

Figure 9. The liquid volume fraction distribution of eight different modified nozzles

The turbulent kinetic energy distribution of eight different modified nozzles. The turbulent kinetic energy distribution of eight different modified nozzles are shown in Fig.10. As can be seen from Fig.10, the turbulent kinetic energy distribution of the 8 modified nozzles are relatively unified. It can be seen that the turbulent kinetic energy is generated by the corner, gradually steady flow in the horizontal tube, and the turbulent kinetic energy of the long nozzle is obviously lower than that of the short nozzle, and the effects of wall area on the stability of flow field is explained from one side. 


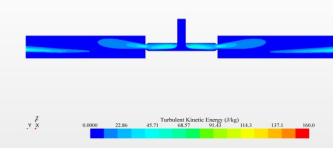

(a)Type 1

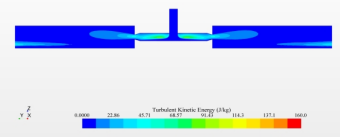

(e)Type 5

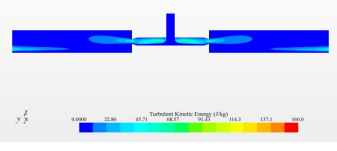

(b)Type 2

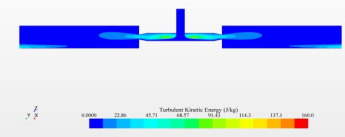

(f)Type 6

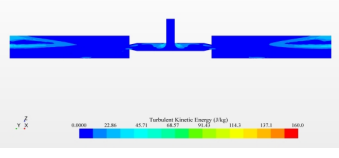

(c)Type 3

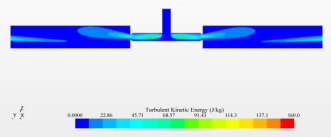

(g)Type 7

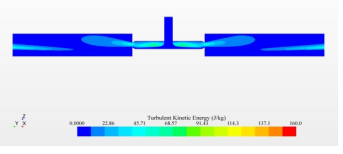

(d)Type 4

Figure 10. The turbulent kinetic energy distribution of eight different modified nozzles

The pressure distribution of eight different modified nozzles. The pressure distribution of eight different modified nozzles are shown in Fig.11. It can be seen from Fig.11, the internal pressure of the eight modified nozzles is almost similar to the prototype. The high pressure zone is produced in the cross section of the vertical tube and the other parts in the tube still have high pressure. So, no matter the diameter of the nozzle has the function to converge the flow field and prevent the flow. The significance of nozzle optimization is also explained.

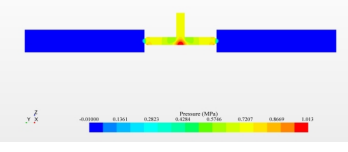

(a)Type 1

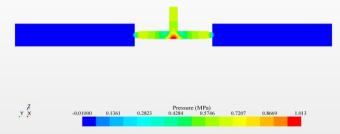

(e)Type 5

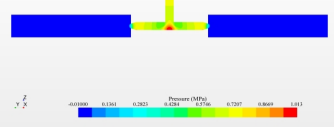

(b)Type 2

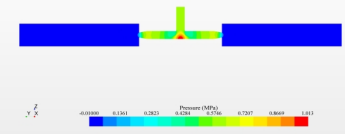

(f)Type 6

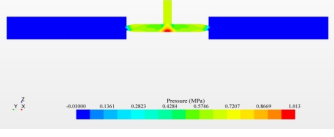

(c)Type 3

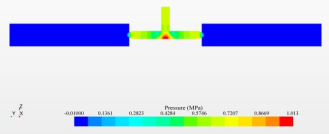

(g)Type 7

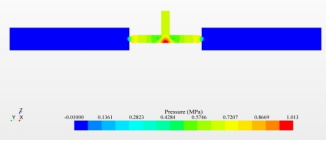

(d)Type 4

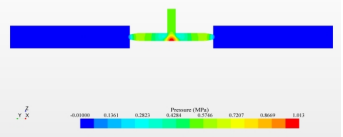

(h)Type 8

Figure 11. The pressure distribution of eight different modified nozzles

\section{Conclusions}

According to the analysis of the above calculation, the type 2 nozzle shows the best performance. Although its mass flow rate and outlet velocity are not the best, but its high turbulence kinetic energy can effectively make up for the lack of outlet velocity.At the same time, after comparing the flow parameters of the 8 type nozzles, we can draw the following conclusions:

1) The acceleration of the long nozzle is better than the short nozzle, but the long nozzle will cause the loss of turbulent kinetic energy, which needs to be considered in the actual equipment selection process.

2) Although large diameter nozzles have high mass flow, but the uneven distribution of velocity in the tube often causes jet skew. 
3) The intersection of vertical tube and horizontal tube bears the maximum pressure, and the change of the nozzle parameters has little influence on the pressure. Some structural strengthening should be done in the production.

Based on the above conclusions, this paper studies the jet flow of nozzles of different diameters and lengths, and selects the nozzle with the best performance of jet flow, providing data support for subsequent design.

\section{References}

[1] Jianhua Zhu. Application of high pressure water cleaning technology in storage tank maintenance 2006 J. Oil and Gas Storage and Transportation, 25(9): 60-62.

[2]Daugllerty R L, Franzini J B. Fluid mechanicas with engineering applications 1985 M. McGraw Hill Companies, 8(1): 485-487.

[3] TingchengLiu, Yu Ding. High pressure water jet cleaning technology 1999 J. Chemical Cleaning, 15(4): 37-42.

[4] Lei Zhuang. Nozzles for high pressure water jet cleaning 2003 J. Cleaning the World, 19(12):28-31.

[5] Huiping Zhou, Kai Wang, Jiangfeng Hu. Practice of on-line tank cleaning for crude oil storage tanks 2011 J. Oil and Gas Storage and Transportation, 30(6): 458-459.

[6] Qinghong Zhang, Zhiqing Fu, Shuting Wan. Study on oil blending rotary nozzle 2012 J. Chemical Equipment and Piping, 49(3): 39-43.

[7]HaihongYang, Blending nozzle application in storage tank 2012 J. Chemical Technology and Development, 41(12): 57-59.

[8] Xiuwen Wang, Renyi Dong. Rotary jet agitator system for oil storage tanks 1997 J. Oil and Gas Surface Engineering, 16(2): 22-23. 